\title{
Re: Transurethral Resection of the Prostate Biopsy of Suspected Anterior Prostate Cancers Identified by Multiparametric Magnetic Resonance Imaging: A Pilot Study of a Novel Technique
}

\author{
Dason S, Allard CB, Wright I, Shayegan B \\ McMaster University Faculty of Medicine, Department of Urology, Ontario, Canada
}

Urology 2016;91:129-135. doi: 10.1016/j.urology.2015.12.063. Epub 2016 Feb 1.

\section{EDITORIAL COMMENT}

miss or undersample anterior prostate cancers (APCs). Multiparametric magnetic resonance imaging (mpMRI) plays an increasing role in the diagnosis of prostate cancer. In this study, the authors report a novel technique of cognitively directed transurethral resection of the prostate (TURP) to biopsy APCs identified by $\mathrm{mpMRI}$ and evaluate the feasibility of this approach among participants with prior negative TRUS-guided biopsies. Participants aged 50-75 years were offered inclusion if they had an elevated prostate-specific antigen level, a lesion suspicious for APC on mpMRI, and at least 1 prior negative TRUS-guided prostate biopsy with a minimum of 10 cores. Exclusion criteria included previous pathologically confirmed prostate cancer, nonanterior prostate lesions, multiple lesions on $\mathrm{mpMRI}$, prostate lesions having benign appearance on $\mathrm{mpMRI}$, and prostate lesions abutting the external urethral sphincter. The TURP biopsy procedure was cognitively directed on the basis of the suspected APC location in 3 dimensions, which had been previously noted from the mpMRI images and the anticipated APC location was resected. A total of 16 consecutive participants were enrolled. Thirteen (81.3\%) participants had clinically significant APC detected by TURP biopsy. Seven participants (43.8\%) underwent radical prostatectomy that confirmed clinically significant disease in all 7 participants. This study shows that among patients with anterior prostate lesions on mpMRI and prior negative TRUS-guided biopsy, TURP biopsy detects some clinically significant cancers and comparative trials on the concept are needed.

Özgür Yaycıoğlu, MD

\section{Re: Comparable Survival of En Bloc versus Standard Donor Kidney Transplants in Children}

\author{
Winnicki E, Dharmar M, Tancredi D, Butani L \\ University of California Davis, Department of Pediatrics, California, USA \\ J Pediatr 2016;19. pii: S0022-3476(16)00131-1. doi: 10.1016/j.jpeds.2016.01.054. Epub ahead of print.
}

\section{EDITORIAL COMMENT}

En bloc kidney transplantation, which refers to harvesting of both kidneys along with major blood vessels, and performing anastomoses of the allograft vena cava and aorta to the recipient vessels, has an increased risk of graft thrombosis and hyperfiltration injury. However, reports of adult recipients from very young donors show very good long-term results. Contrary to that in adults, the data on en bloc kidneys in pediatric recipients is limited. In this retrospective cohort study, the authors has compared time to allograft failure and estimated glomerular filtration rate (eGFR) in 6882 pediatric recipients of both en bloc and standard criteria deceased donors using Organ Procurement and Transplantation Network data for over a 13-year period. Besides showing a similar allograft survival to standard criteria deceased donors, en bloc kidney recipients (1.8\%) also had a shorter wait list time and superior kidney function in the intermediate term supporting the use of these kidneys for pediatric recipients. The use of en bloc pediatric kidney transplants is a reasonable option for children awaiting a cadaveric kidney at transplantation centers experienced in en bloc kidney transplantation. However, longer-term outcomes, including the risk of hyperfiltration injury, need to be determined. 